\title{
ECG Denoising Using Mutual Information Based Classification of IMFs and Interval Thresholding
}

\author{
Marjaneh Taghavi, Mohammad B. Shamsollahi, and Lotfi Senhadji
}

\begin{abstract}
The Electrocardiogram (ECG) is widely used for diagnosis of heart diseases. Therefore, the quality of information extracted from the ECG has a vital role. In real recordings, ECG is corrupted by artifacts such as prolonged repolarization, respiration, changes of electrode position, muscle contraction, and power line interface. In this paper, a denoising technique for ECG signals based on Empirical Mode Decomposition (EMD) is proposed. We use Ensemble Empirical Mode Decomposition (EEMD) to overcome the limitations of EMD. Moreover, to overcome the limitations of thresholding methods we use the combination of mutual information and two EMD based interval thresholding approaches. Our new method is evaluated on ECG signals available in MIT-BIH database. This method is compared with two EEMD based interval thresholding methods. The results show that our proposed method has a better Signal to Noise Ratio improvement (SNRimp) and a lower Mean Square Error (MSE) than the other two methods.
\end{abstract}

Keywords-Ensemble Empirical Mode Decomposition; denoising; Mutual Information; Instantaneous Half Period; Interval Thresholding; ECG

\section{INTRODUCTION}

$\mathbf{E}$ LECTROCARDIOGRAM (ECG) signal is a recording of the electric potential produced by rhythmical cardiac activities. ECG is widely used in clinical applications where the accuracy of information from ECG is very important. In [1]-[4], numerous methods have been proposed to denoise ECG signal. Empirical Mode Decomposition (EMD) is a typical tool for denoising signals, since EMD is very suitable for nonlinear and nonstationary signals. EMD was initially introduced by Huang [1], and immediately used in biomedical engineering applications [5], [6]. EMD decomposes a signal into several Intrinsic Mode Functions (IMFs). The EMD denoising is based on the fact that noise components are often found in the first several IMFs. Thus, denoised signal is obtained by detecting noisy IMFs and removing or filtering them and then the signal is reconstructed by filtered IMFs.

EMD has some limitations in decomposing signal which has the close amplitude-frequency ranges to each other. This problem is termed 'mode mixing', and it can be happened

Manuscript Manuscript received February 28, 2014.

M. Taghavi is with the School of Engineering and Science, Sharif University of Technology, International Campus Kish Island, Iran. (e-mail: mtaghavi@kish.sharif.edu).

M. B. Shamsollahi is with the Biomedical Signal and Image Processing Laboratory (BiSIPL), School of Electrical Engineering, Sharif University of Technology, Tehran, Iran. (e-mail: mbshams@sharif.edu).

L. Senhadji is with the Laboratoire Traitement du Signal et de l'Image (LTSI), Université de Rennes 1, Rennes, France. (e-mail: lotfi.senhadji@univ-rennes1.fr). when different modes of oscillation are appeared in a single IMF. We use Ensemble Empirical Mode Decomposition (EEMD) from [7] to solve this problem. In EEMD, an ensemble mean is taken over a number of IMFs extracted from EMD applied to some new noisy data obtained by adding a white noise series each time to the original data. Numerous methods such as soft and hard thresholding are proposed to filter noisy IMFs [8]-[10]. In these methods it is assumed that all IMFs are noisy and thresholding operations are applied to them. Hence, some useful information is lost especially in higher order IMFs which contain more signal information than noise.

In this paper, we combine two EMD based interval thresholding, mutual information technique and correlation coefficient test for ECG denoising. EEMD is applied to the noisy signal to obtain IMFs and then mutual information is used to classify IMFs into high frequency (HF) and low frequency (LF) groups [11]. We consider HF group as a strong noisy IMFs. Then we use Block Energy (BE) model [12] to filter HF group. Some of IMFs in the LF group may have dominant frequency component of the original signal. Therefore, we use correlation coefficient test to detect noise free IMFs [13] and then we filter the noisy IMFs of LF group by Instantaneous Half Period model (IHP) model [14]. Finally, the denoised signals are reconstructed by adding filtered IMFs from LF and HF groups to the remaining nonfiltered IMFs which are in the LF group. Therefore in this method the useful information of signal in each IMF can be preserved. In order to demonstrate the performance of our proposed method, we compare its performance with IHP model and BE model [12] in terms of improvement in signal to noise ratio $\left(\mathrm{SNR}_{\mathrm{imp}}\right)$ and Mean Square Error (MSE). For this comparison, we have used different type of ECG signals from the MIT-BIH database.

The outline of the paper is as follows. In section II, a brief review of theoretical EMD, EEMD, BE model, IHP model and mutual information are presented. Furthermore, our proposed method is explained. Section III illustrates the results of the three methods and their performance will be compared. Finally, conclusion is made in section IV.

\section{METHODOLOGY}

\section{A. Description of EMD and EEMD}

EMD is able to decompose a given signal into sum of IMFs by iterative sifting process. An IMF should satisfy the following two conditions: (1) the number of extrema and the number of zero crossings are either equal or differ at most by one; and (2) the mean of its upper and lower envelopes equals zero [1]. The sifting process for extracting an IMF from a given signal $x(t)$ is described as follows: 
1. Detect all local maxima and minima of signal $x(t)$;

2. Use cubic spline line to interpolate between maxima of $x(t)$ to get its upper envelop, $e_{u p}(t)$ and similarly to interpolate between minima to get its lower envelop, $e_{\text {low }}(t)$

3. Calculate the average between those two envelopes and extract the details:

$d(t)=x(t)-\left[e_{\text {up }}(t)+e_{\text {low }}(t)\right] / 2$

The above procedure to extract the IMF is called the sifting process.

4. Repeat step 1 to 3 until the resulting signal, $d(t)$, has two conditions of IMF, then the first IMF, $c_{1}(t)$, is obtained. There are some criteria for terminating the sifting process. A commonly used one is the Standard Deviation (SD):

$$
\sum_{t=0}^{T} \frac{\left|d_{k-1}(t)-d_{k}(t)\right|^{2}}{d_{k-1}^{2}(t)} \leq S D
$$

where $\mathrm{k}$ is the number of sifting iteration. The value of SD is usually in the range $[0.2,0.3]$.

5. Treat the residue signal $r_{1}=x(t)-c_{1}(t)$ as a new signal and apply the sifting process again;

6. Repeat step 1 to 5 to obtain IMFs until the residue of signal, step 5, is constant. The signal $x(t)$ is expressed as:

$$
x(t)=r_{I}+\sum_{i=1}^{I} c_{i}(t)
$$

where $\mathrm{I}$ is the number of IMFs and $r_{I}$ is the final residue. There are a number of considerations used in implementing EMD such as number of sifting, stop criteria and envelop interpolation [1], [15]. As an improved version of EMD, EEMD was originally developed to reduce the mode-mixing effect [7]. In this method white noise is added to the original signal for many times with different series. Then average IMFs are obtained by calculating mean of IMFs which are obtained by applying EMD on each trial, the final IMFs are:

$$
\bar{c}_{i}(k)=\frac{1}{M} \sum_{m=1}^{M} c_{i, m}(k) \quad i=1,2,3, \ldots, I
$$

where I is the number of IMFs and M is the number of trials. The added noises are different in each trial and their effect can be cancelled out on average, under the condition that $\mathrm{M}$ is high enough [5].

\section{B. EMD Based denoising using interval thresholding}

In this section, we study two methods to detect noisy parts of IMFs and then filter them to obtain denoised IMFs.

\section{1) Instantaneous Half Period (IHP)}

In [14] a criterion is proposed to detect the noisy parts of IMFs based on the time interval between two zero-crossings of each IMF. In this method, the noisy parts are detected if the time interval between two zero-crossings is smaller than predefined threshold. Then, the noisy parts are removed and the signal is reconstructed by remaining parts of IMFs [14]. Steps of this method are as follows:

1. Find zero crossings of each IMF;

2. Obtain time interval between two adjacent zero-crossings of each IMF;
3. Detect noisy parts of each IMF as follows and replace these part with zero:

$$
\hat{c}_{i}(k)=\left\{\begin{array}{c}
c_{i}(k), T_{i}^{j} \geq t h r \\
0, \text { otherwise }
\end{array}, Z P_{i}^{j}<k \leq Z P_{i}^{j+1}\right.
$$

4. Denoised signal can be obtained as:

$$
\hat{x}(t)=r_{I}+\sum_{i=1}^{I} \hat{c}_{i}(t)
$$

where $\hat{x}(t)$ is the denoised signal, $\hat{c}_{i}(t)$ is the $i^{t h}$ denoised IMF, $i=1,2,3, \ldots . . I, I$ is the number of IMFs. $Z P_{i}^{j}$ is $j^{t h}$ zero-crossing of $i^{\text {th }}$ IMF. $\tau_{i}^{j}$ is the time when $j^{\text {th }}$ zero crossing of $i^{\text {th }}$ IMF emerges. $T_{i}^{j}$ is the time interval between two zero-crossings of $i^{\text {th }} \mathrm{IMF}, T_{i}^{j}=\tau_{i}^{j+1}-\tau_{i}^{j}$. In this method, finding the value of threshold is very important. The optimum threshold is obtained based on the maximum frequency of signal [14]:

$t h r_{o p t}=\frac{\alpha}{2 f_{h}}$

where $f_{h}$ denotes the maximum frequency of the signal and $\alpha$ is the constant coefficient used to determine the frequency range of the retained oscillation [14].

\section{2) Block Energy Model}

In [12], an interval thresholding method is proposed to detect noisy parts of IMFs based on the energy of samples which are between two zero-crossings of each IMF. The threshold value is determined for each IMF. The waveform between the two adjacent zero crossings of IMF is retained when the energy of samples which are between the two adjacent zero crossings is larger than the predefined threshold, whereas the waveform is set to zero. Steps of interval thresholding are as follows:

1. Find zero crossings of each IMF;

2. Determine the samples which are between the two adjacent zero crossing of IMF:

$Q_{i}^{j}=\left\{q_{i}^{1}, q_{i}^{2}, q_{i}^{3}, \ldots q_{i}^{k_{j}}\right\}$

where $Q_{i}^{j}$ is the sample points set between two adjacent zero crossing of $i^{\text {th }} \mathrm{IMF}\left[p_{i}^{j}, p_{i}^{j+1}\right]$ and $k_{j}$ is the number of sample points.

3. Determine the energy of each sample point set:

$$
S_{i}^{j}=\sum_{k=1}^{k=k_{j}}\left|c_{i}\left(q_{i}^{k}\right)\right|^{2}, q_{i}^{k} \in Q_{i}^{j}
$$

4. Determine threshold value for each IMF by using equation (13);

5. Detect noisy parts of each IMF and filter it as follows:

$$
\hat{c}_{i}\left(Q_{i}^{j}\right)=\left\{\begin{array}{c}
c_{i}\left(Q_{i}^{j}\right)\left(1-\frac{T_{i}}{S_{i}^{j}}\right) S_{i}^{j} \geq T_{i} \\
0 \quad S_{i}^{j}<T_{i}
\end{array}\right.
$$

where $\hat{c}_{i}\left(Q_{i}^{j}\right)$ denotes the $i^{\text {th }}$ filtered IMF, $S_{i}^{j}$ is the energy of sample point set between $j^{\text {th }}$ and $j+1^{\text {th }}$ zero crossings of $i^{t h}$ IMF, $T_{\mathrm{i}}$ is the threshold value for $i^{\text {th }} \mathrm{IMF}$. The denoised signal is obtained by using question (6).

In [16] a denoising method based on the IMF energies is proposed. The IMF energy should be an exponentially 
decreasing function of the IMF index [16]. Therefore the first IMF has the highest amount of energy. As in this paper we consider white Gaussian noise, the noise-only IMF energies for $k \geq 2$ can be approximated as a function of the first IMF energy [16] according to

$E_{k}=\left(\frac{E_{1}}{\beta}\right) \rho^{-2(1-H) k}$

where $E_{1}$ is the energy of the first IMF, $\rho$ is a scaling factor and is estimated as $\rho \approx 2$ [16], $\beta$ is experimentally estimated in [16] and $\mathrm{H}$ is the Hurst exponent as for specific EMD implementation [16]. The energy equation of the first IMF is given:

$E_{1}=\frac{1}{N} \sum_{n=1}^{N}\left(I M F_{1}(n)\right)^{2}$

The optimal value of threshold in this method is suggested in [12]:

$T_{i}=\frac{c V_{i} \sqrt{2 \log _{2}(N)}}{N}$

where $T_{i}$ is the determined threshold value for $i^{\text {th }} \mathrm{IMF}, \mathrm{c}$ is a constant experimentally found to take values 1 to $0.7, V_{i}$ is the noise variance of $i^{\text {th }}$ IMF which is calculated in (11) for $i>1$ and $\mathrm{N}$ is the number of IMF samples. We assume that most of the noise energy is in the first IMF [17], so we use the absolute median deviation of the first IMF for $V_{l}$ as in [18].

$V_{1}=\left(\frac{\text { median }\left(\mid I M F_{1}-\text { median }\left(I M F_{1}\right) \mid\right)}{0.6745}\right)^{2}$

\section{IMF classification based on Mutual information}

Mutual information quantifies the relationship between two random variables [19]. Mutual information has been used in different applications such as feature selection [20] and signal denoising [11]. The mutual information is used in EMD based denoising for separating IMFs into high frequency and low frequency groups. Steps of classification of IMFs based on mutual information are as follows [11]:

1. The initial partition index (IPI) is determined by [Num/2]. Num is the number of IMFs, considering residue, and [ ] denotes the integer part. The sum of all IMFs whose index is greater than IPI is called the initial LF group and the sum of the remaining IMFs is called initial HF group;

2. Compute mutual information between noisy signal and IMF which has [Num/2] index. Then add IMFs which have $[\mathrm{Num} / 2]+1$ and $[\mathrm{Num} / 2]$ index. Then compute mutual information between this new signal set and noisy signal. Repeat this procedure. Therefore, the IMFs in the LF group are iteratively added and the new set signal is obtained and then the mutual information between noisy signal and the new signal set is computed. This act is done until all the modes have been added. Consider residue as an IMF as well.

3. The final partition index $(F)$ is set as the index of IMF with minimum mutual information;

4. The new HF group is the sum of IMFs with index less than $F$. The sum of IMFs with equal or greater than $F$ is the final LF group:

$x(t)=\sum_{i=1}^{F-1} c_{H i}(t)+\sum_{i=F}^{I} c_{L i}(t)$ where $x(t)$ is the original signal, $c_{H i}(t)$ and $c_{L i}(t)$ are the $i^{\text {th }}$ IMF in the HF and LF groups, I is the number of IMFs and residue is concidered as an IMF in the LF group.

\section{Proposed method}

In this paper, we propose a denoising method based on the combination of EMD based interval threshoding, mutual information and correlation coefficient test. EEMD is applied to the noisy ECG signal to decompose it into several IMFs. Then we use mutual information to classify IMFs into high frequency and low frequency groups as explained in section C. We use BE model to filter HF group as in section B. In this interval thresholding, a determined threshold value for each IMF is defined in order to gain more accuracy and performance. Moreover it is possible to guess the samples are either noise or signal, based on the energy of samples. Whereas based on the absolute amplitude of the $i^{\text {th }}$ IMF, it is impossible to know whether samples are noise or not [12]. The IMFs in LF group contain low frequencies of the original signal or noise or both of them. Therefore, we use correlation coefficient test (c.c) to detect IMFs with dominant frequency of the original signal [13]

(c. c $)_{j}=\frac{1}{\sqrt{E_{x} E_{c_{j}}}} \sum_{i=1}^{N} X\left(f_{i}\right) C_{j}\left(f_{i}\right)$

where $(c . c)_{j}$ is the correlation coefficient between $j^{\text {th }}$ IMFs $\left(c_{j}\right)$ and noisy signal $x(t)$ in frequency domain, $E_{x}$ and $E_{c_{j}}$ are their total energies, $X\left(f_{i}\right)$ and $C_{j}\left(f_{i}\right)$ are their DFTs and $N$ is the number of data points.

$E_{x}=\sum_{i=1}^{N}\left|X\left(f_{i}\right)\right|^{2}, \quad E_{\left(c_{j}\right)}=\sum_{i=1}^{N}\left|C_{j}\left(f_{i}\right)\right|^{2}$

The correlation coefficient ranges from values +1 to -1 . If the absolute value of correlation coefficient of IMFs in the LF group is greater than 0.2 , it indicates that the IMFs have dominant frequency component of the original signal [13]. The remaining IMFs contain noise component and then should be filtered. The frequency range of signal is often lower than the frequency of noise. Therefore the time interval between two adjacent zero crossings of signal dominated oscillation is longer than noise dominated oscillation [14]. So we use IHP model to filter noisy LF IMFs as explained in section B. Finally, the denoised signal is obtained by adding filtered IMFs from two interval thresholding output and the remaining non-filtered IMFs in LF group. The flowchart of the proposed algorithm is shown in Fig. 1.

\section{RESULTS}

Our method is applied to the several standard data sets from PhysioBank [21], including MIT-BIH Arrhythmia database (DB1) [22], MITBIH normal sinus rhythm database (DB2) [23] and the MIT-BIH noise stress test database (DB3) [24]. Ten records are arbitrarily chosen from DB1. Each signal contains 60 seconds recording and is digitized at 360 $\mathrm{Hz}$. The first 60 seconds of all 18 records of DB2 are used too. White Gaussian noises are added artificially to the ECG signals to make $-5 \mathrm{~dB}, 0 \mathrm{~dB}, 5 \mathrm{~dB}$, input SNRs. The performance of the proposed method is evaluated by 
improvement of signal to noise ratio $\left(S N R_{\text {imp }}\right)$ and mean square error (MSE). For each input SNR, 100 times runs are performed to get an averaged $S N R_{\text {out }}$ and MSE value. The $S N R_{i m p}$ can be represented as follows:

$S N R_{\text {imp }}=S N R_{\text {out }}-S N R_{\text {in }}$

where $S N R_{\text {out }}$ and $S N R_{\text {in }}$ are formulated as follows:

$S N R_{\text {in }}=10 \times \log \left(\frac{\sum_{n=0}^{L-1} x(n)^{2}}{\sum_{n=0}^{L-1}(y(n)-x(n))^{2}}\right)$

$S N R_{\text {out }}=10 \times \log \left(\frac{\sum_{n=0}^{L-1} x(n)^{2}}{\sum_{n=0}^{L-1}(\hat{x}(n)-x(n))^{2}}\right)$

where $x(n)$ is the clean ECG signal, $y(n)=x(n)+v(n)$ is the noisy signal, $\hat{x}(n)$ is the denoised signal and $v(n)$ is the noise. We have also used MSE criterion for evaluating quality of the information which is preserved in the denoised signal, it can be represented as follows:

$M S E=\frac{\sum_{n=0}^{L-1}(\hat{x}(n)-x(n))^{2}}{L}$

where L is the length of the signal. Fig. 2 shows an example of a clean signal, noisy signal and denoised signal using our proposed method. The tested signal in Fig. 2 is the record 100 from DB1 with an additive Gaussian noise that makes a $0 \mathrm{~dB}$ input SNR. We apply EEMD on this record to obtain IMFs. The total number of IMFs is 15 considering residue as well. Then we use mutual information to classify IMFs into HF and LF group. The IPI index is 7 and the $F$ index is 9 . So the sum of 8 first components $\left(\mathrm{IMF}_{1}\right.$ to $\left.\mathrm{IMF}_{8}\right)$ gives the final HF group and the sum of remaining IMFs gives the final LF group. Then we calculate the correlation coefficient to detect the IMFs in the LF group which should be filtered. For this signal, the IMFs $9,12,14,15$ are obtained. So the remaining components in LF group ( $\mathrm{IMF}_{10}, \mathrm{IMF}_{11}$ and $\left.\mathrm{IMF}_{13}\right)$ need to be filtered. We use BE model to filter IMFs in the HF group with a determined threshold for each IMFs and IHP model is applied to $\mathrm{IMF}_{10}, \mathrm{IMF}_{11}$ and $\mathrm{IMF}_{13}$. Finally we add filtered IMFs and the IMFs with index 9, 12, 14, 15 to obtain denoised signal as shown in Fig. 2.

Table I shows the values for output SNR for different values of input SNR of the ECG records of DB1 for three methods: our proposed method, EEMD-based IHP model (IHP) [14] and EEMD-based BE model [12]. We can compare their performance in SNR improvement from (18).

As can be seen in Table I, in all three methods, by increasing the input SNR, ECG signal becomes cleaner and therefore the SNR improvement is lower. From Table I, it is clear that our proposed method has better performance than the other two methods for all inputs SNR (i.e.-5, 0, 5, dB) and all ECG records.

Table II shows the average values of MSE for ten ECG records from DB1 as in Table I. We can see the denoised signal which is obtained by our method has a less error than two other methods. We plot the output SNR versus different input SNRs for all 18 records of DB2 in Fig. 3. The IHP and $\mathrm{BE}$ model assume that the noise in the signal is spread over all IMFs and the threshold value is determined for each IMF. Therefore, some useful information may be lost, especially when input SNR is increased. We can see the BE model has larger output SNR than IHP model.

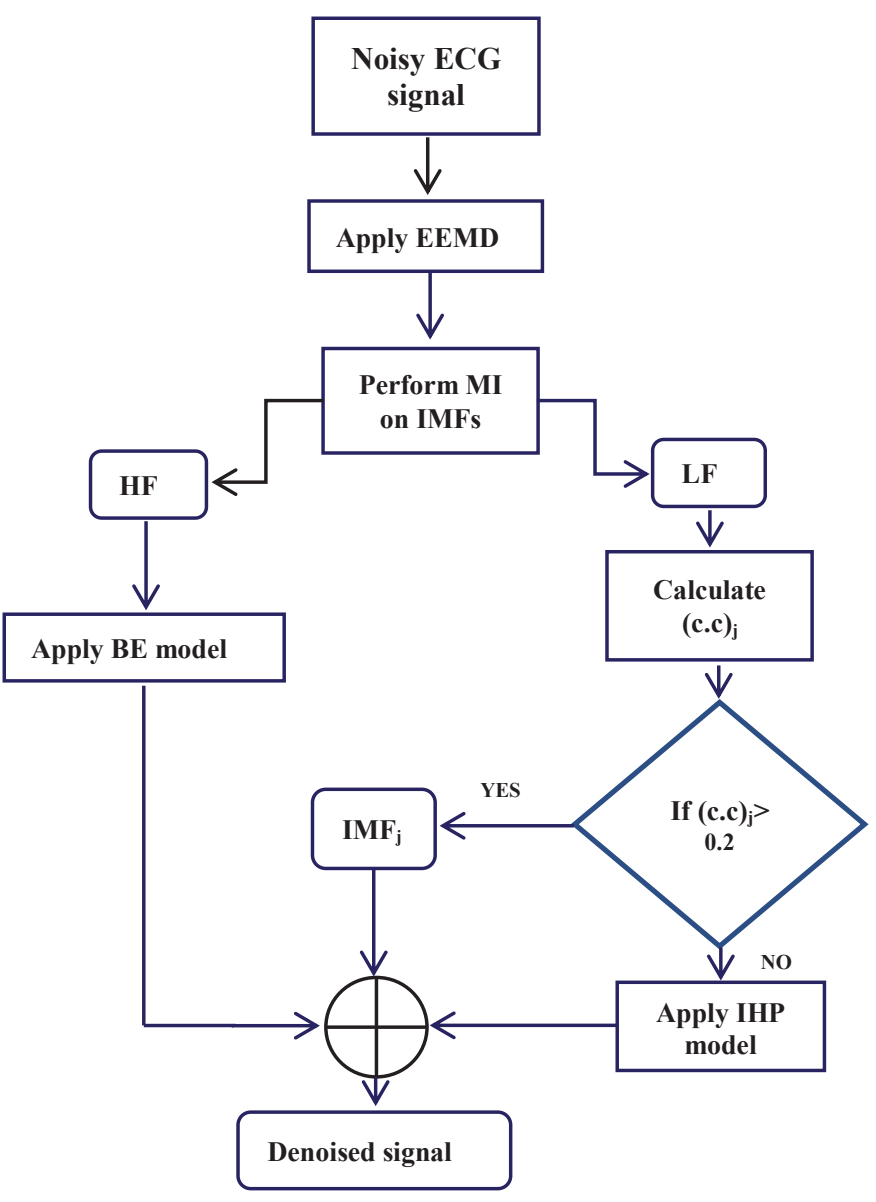

Fig. 1. The flowchart of the proposed algorithm for performing denoising of a given signal.

Therefore, according to the (18), the BE model has a better $\mathrm{SNR}$ improvement. It is clear that BE model has more accuracy in detecting noisy parts of IMFs than the IHP model. So, we use BE model to filter IMFs in the HF group which contain more noise components and we use IHP model to filter noisy IMFs in the LF group to preserve signal information.
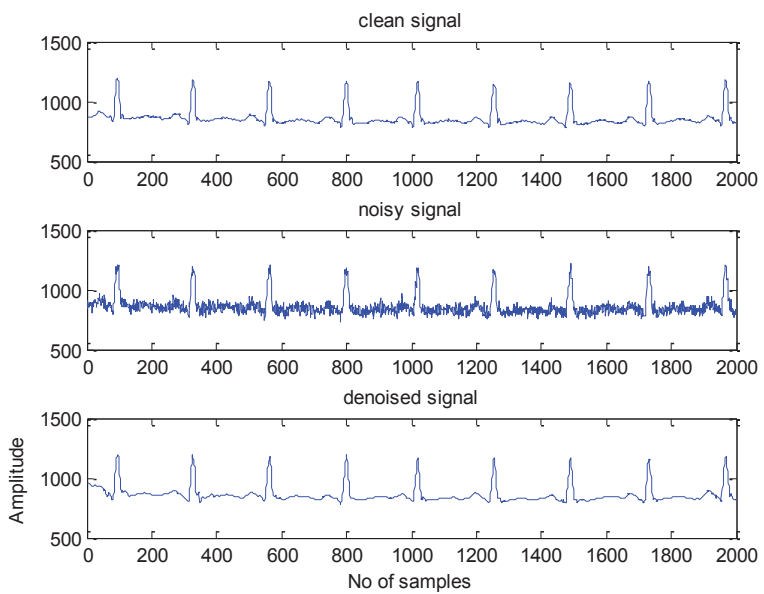

Fig. 2. Clean ECG, Noisy ECG with $5 \mathrm{~dB}$ SNR and ECG denoised by the proposed algorithm 
TABLE I

OUTPUT SNR FOR PROPOSED METHODS, IHP MODEL AND BE MODEL

\begin{tabular}{|c|c|c|c|c|c|c|c|c|c|c|}
\hline \multirow{2}{*}{\multicolumn{2}{|c|}{$\begin{array}{c}\text { Input SNR } \\
\text { Algorithm }\end{array}$}} & \multicolumn{3}{|c|}{$-5 \mathrm{~dB}$} & \multicolumn{3}{|c|}{$0 \mathrm{~dB}$} & \multicolumn{3}{|c|}{$5 \mathrm{~dB}$} \\
\hline & & IHP & $\mathrm{BE}$ & Proposed & IHP & BE & Proposed & IHP & BE & Proposed \\
\hline \multirow{10}{*}{ 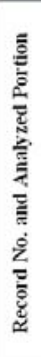 } & 100 & 9.79 & 10.23 & 10.95 & 11.43 & 13.78 & 14.08 & 13.01 & 15.87 & 16.51 \\
\hline & 111 & 10.13 & 11.04 & 11.56 & 11.72 & 14.23 & 14.58 & 15.29 & 15.67 & 16.06 \\
\hline & 113 & 8.02 & 10.43 & 11.13 & 9.56 & 12.71 & 14.31 & 9.98 & 14.04 & 16.21 \\
\hline & 115 & 7.59 & 10.63 & 10.95 & 9.27 & 13.07 & 13.45 & 9.95 & 14.19 & 15.57 \\
\hline & 116 & 7.37 & 10.08 & 10.15 & 9.95 & 12.61 & 13.46 & 11.10 & 14.91 & 15.96 \\
\hline & 117 & 9.83 & 10.95 & 11.21 & 12.44 & 13.55 & 14.31 & 13.80 & 15.04 & 16.53 \\
\hline & 121 & 8.31 & 10.01 & 11.64 & 9.55 & 11.65 & 13.97 & 10.05 & 12.61 & 16.15 \\
\hline & 123 & 9.13 & 11.38 & 11.65 & 11.51 & 14.08 & 14.79 & 12.79 & 14.93 & 16.05 \\
\hline & 124 & 7.15 & 11.79 & 12.54 & 7.92 & 14.83 & 15.78 & 9.70 & 17.25 & 18.17 \\
\hline & 231 & 7.04 & 11.16 & 11.39 & 8.07 & 11.16 & 14.65 & 9.50 & 16.19 & 17.28 \\
\hline \multicolumn{2}{|c|}{ Average } & 8.23 & 10.77 & 11.31 & 10.14 & 13.16 & 14.34 & 11.51 & 15.07 & 16.45 \\
\hline
\end{tabular}

TABLE II

PERFORMANCE EVALUATINO OF PROPOSED MTHODS, IHP MODEL AND BE MODEL BASED ON MSE

\begin{tabular}{|c|c|c|c|}
\hline \multirow{2}{*}{ Algorithm } & \multicolumn{3}{|c|}{ Input SNR (dB) } \\
\cline { 2 - 4 } & $\mathbf{- 5}$ & $\mathbf{0}$ & $\mathbf{5}$ \\
\hline Proposed & 0.091 & 0.064 & 0.036 \\
\hline BE & 0.112 & 0.075 & 0.047 \\
\hline IHP & 0.147 & 0.092 & 0.085 \\
\hline
\end{tabular}

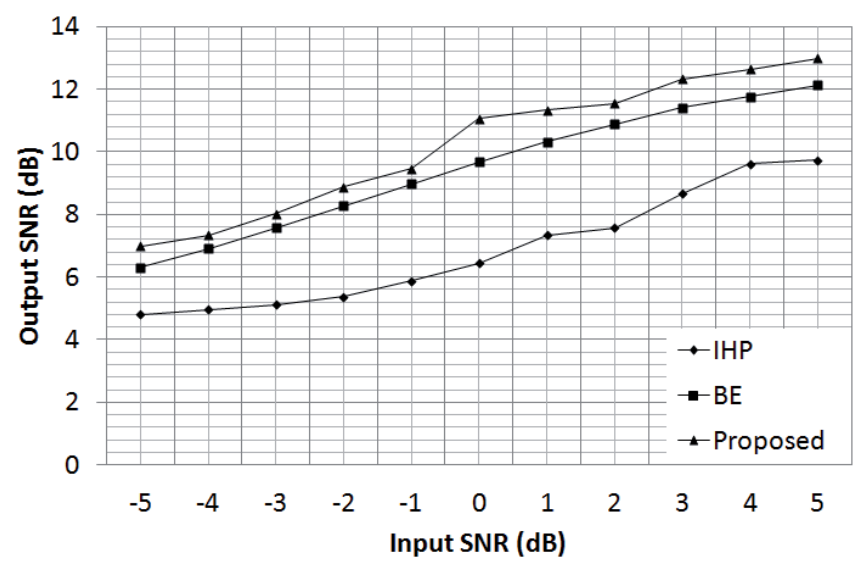

Fig. 3. Output SNR versus different input SNRs for the first 60 seconds of all 18 records of DB2.

In our proposed method all IMFs are filtered with minimum loss of useful information, and maximum noise removal is achieved in comparison to the single method of IHP model or BE model.

This is obtained by using mutual information, correlation coefficient test, and two different interval thresholding approaches. So, this Model has more accuracy in recognizing and filtering noisy IMFs. The performance of our proposed method is evaluated by real noise. Fig. 4 shows denoising results for different input ECG records with an additive EMG noise. The input SNR of records 118e 24 and 118e18 from DB3 is $6 \mathrm{~dB}$ and for record 116 from $\mathrm{dB} 1$ is $10 \mathrm{~dB}$. The output SNRs which are obtained by applying our method is $16.7 \mathrm{~dB}$ for $118 \mathrm{e} 24,20.4 \mathrm{~dB}$ for 116 and $17.4 \mathrm{~dB}$ for $118 \mathrm{e} 18$. As it can be seen, the denoised signal follows the clean ECG morphology.

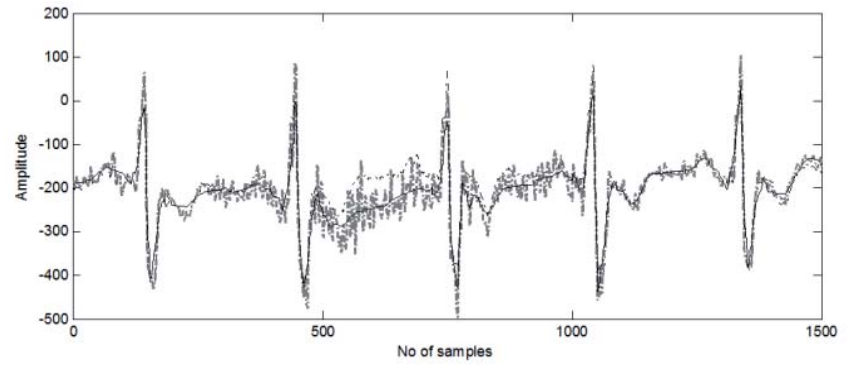

(a)

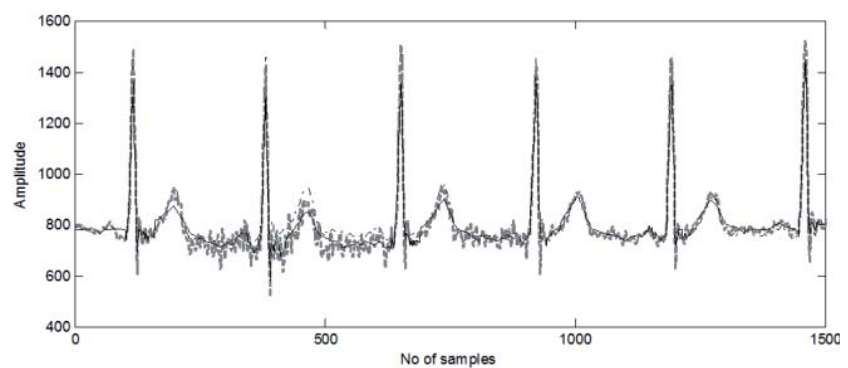

(b)

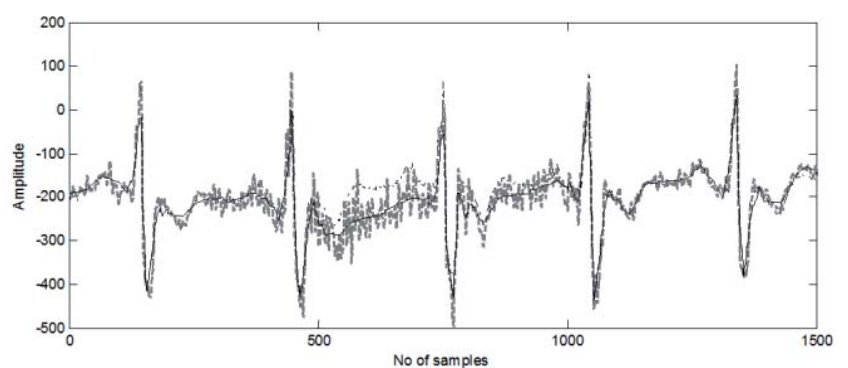

(c)

Fig. 4. Typical filtering results of proposed method for different input ECGs with an additive real EMG noise. Dash-dot is the clean signal, solid line is the denoised signal and dash line is the nosiy signal. (a) Record $118 \mathrm{e} 24$ from dB3 (corresponding to an input SNR of 6dB). (b) Record 116 from dB1 (corresponding to an input SNR of $10 \mathrm{~dB}$ ). (c) Record 118e18 from DB3 (corresponding to an input SNR of $6 \mathrm{~dB}$ ).

\section{CONCLUSION AND DISCUSSION}

This paper presents a method based on EEMD to reduce noise from corrupted ECG signals. In this method, EEMD is used to resolve mod mixing problem of EMD. Moreover, two interval thresholding approaches in combination with mutual information and correlation coefficient test are used to preserve signal information. Our method provides a larger SNR improvement, especially for fewer input SNRs, where the original signal is lost in noise. The denoised signal obtained by our method follows better the original signal and has a less MSE than the methods based on IHP or BE model. Thus, by using our method, the noise components will be removed from the signal with minimum loss of useful information in all input SNRs.

\section{REFERENCES}

[1] N. E. Huang, Z. Shen, S.R. Long, M.C. Wu, H.H. Shih, Q. Zheng, N.C. Yen, C.C. Tung, H.H. Liu, "The empirical mode decomposition and the Hilbert spectrum for nonlinear and nonstationary time series analysis", Proc. R. Soc. London., vol. 454, pp. 903-995, 1998. 
[2] A. K. Barros, A. Mansour, and N. Ohnishi, "Removing artifacts from electrocardiographic signals using independent component analysis,' Neurocomputing, vol. 22, pp. 173-186, Nov. 1998.

[3] S. Poornachandra, "Wavelet-based denoising using subband dependent threshold for ECG signals," Digital signal processing, vol.18, pp. 49-55, Jan. 2008.

[4] N. V. Thakor and Y. S. Zhu, "Application of adaptive filtering to ECG analysis: Noise cancellation and arrythmia detection," IEEE Trans. Biomed. Eng., vol. 38, no. 8, pp. 785-794, Aug. 1991.

[5] W. Huang, Z. Shen, N.E. Huang, Y.C. Fung, "Engineering analysis of biological variables: an example of blood pressure over 1 day," Proc. Nat. Acad. Sci. USA, vol. 95, no. 9, pp. 4816-4821, 1998.

[6] H. Liang, Z. Lin, R.W. McCallum, "Artifact reduction in electrogastrogram based on the empirical model decomposition method", Medical \& Biologicl Engineering \& Computing, vol. 38, pp. $35-41.2000$

[7] Z. Wu, N.E. Huang., "Ensemble empirical Mode decomposition: a noise-assisted data analysis method," Advances in Adaptive Data Analysis, vol. 1, no. 1, pp. 1-41, 2009.

[8] A.O. Boudraa, J.C. Cexus, Z. Saidi, "EMD-based signal noise reduction". Int.J. Signal Process, 1, 33-37, 2004.

[9] J. H. Xi and J. Chen, "Empirical Mode Decomposition De-Noising Method Based on Improved Soft-Threshold," Advanced Engineering Forum, vol. 1, pp. 421-425, Sep. 2011.

[10] L. She, Z. Xu, S. Zhang, and Y. Song, "De-noisng of ECG based on EMD improved-thresholding and mathematical morphology operation," 2010 3rd International Conference on Biomedical Engineering and Informatics, vol. 2, pp. 838-842, Oct. 2010.

[11] O. A. Omitaomu, V. A. Protopopescu, and A. R. Ganguly, "Empirical Mode Decomposition Technique With Conditional Mutual Information for Denoising Operational Sensor," vol. 11, no. 10, pp. 2565-2575, 2011.

[12] X. Zhang, Yi Shen, Naizhang Feng, and Zhenhua Wang, "An Empirical Mode Decomposition Signal Denoising Method Based on Block Energy," International Journal of Advancements in Computing Technology, vol. 4, no. 4, pp. 226-234, Mar. 2012.
[13] D. Siwal, V. Suyal, A. Prasad, S. Mandal, and R. Singh, "A new approach of denoising the regular and chaotic signals using Empirical Mode Decomposition: comparison and application.," The Review of scientific instruments, vol. 84, no. 7, p. 075117, Jul. 2013.

[14] Y.-M. Fang, H.-L. Feng, J. Li, and G.-H. Li, "Stress wave signal denoising using ensemble empirical mode decomposition and an instantaneous half period model.," Sensors (Basel, Switzerland), vol. 11, no. 8, pp. 7554-67, Jan. 2011.

[15] G. Rilling., P. Flandrin. and P. Gonçalvès., "On empirical mode decomposition and its algorithms", IEEE-EURASIP Workshop on Nonlinear Signal and Image Processing NSIP- 03, Grado (I), 2003.

[16] P. Flandrin, G. Rilling, and P. Gonçalvès, "EMD equivalent filter banks, from interpetation to applications", Hilbert-Huang Transform and Its Application, 2005.

[17] G. Tsolis and T. D. Xenos, "Signal Denoising Using Empirical Mode Decomposition and Higher Order Statistics," vol. 4, no. 2, pp. 91-106, 2011.

[18] A. O. Boudraa, and J. C. Cexus, "Denoising via empirical mode decomposition, " in Proc. ISCCSP 2006, 2006.

[19] Cover, Thomas M., and Joy A. Thomas. Elements of information theory. John Wiley \& Sons, 2012.

[20] F. Fleuret, "Fast binary feature selection with conditional mutual information,"J. Mach. Learning Res., vol. 5, pp. 1531-1555, 2004.

[21] PhysioBank, physiologic signal archives for biomedical research [Online]. Available: http://www.physionet.org/physiobank/

[22] The MIT-BIH Arrhythmia Database [Online]. Available at: http://www.physionet.org/physiobank/database/mitdb/

[23] The MIT-BIH Normal Sinus Rhythm Database [Online]. Available at: http://www.physionet.org/physiobank/database/nsrdb/

[24] The MIT-BIH Noise Stress Test Database [Online]. Available at: http://www.physionet.org/physiobank/database/nstdb/ 